\title{
Corrigendum to "Identification of Patients Affected by Mitral Valve Prolapse with Severe Regurgitation: A Multivariable Regression Model"
}

\author{
Paola Songia, ${ }^{1,2}$ Benedetta Porro, ${ }^{1}$ Mattia Chiesa, ${ }^{1}$ Veronika Myasoedova, ${ }^{1}$ \\ Francesco Alamanni, ${ }^{1,3}$ Elena Tremoli, ${ }^{1}$ and Paolo Poggio ${ }^{1}$ \\ ${ }^{1}$ Centro Cardiologico Monzino IRCCS, Milan, Italy \\ ${ }^{2}$ Dipartimento di Scienze Farmacologiche e Biomolecolari, Università degli Studi di Milano, Milan, Italy \\ ${ }^{3}$ Dipartimento di Scienze Cliniche e di Comunità, Università degli Studi di Milano, Milan, Italy \\ Correspondence should be addressed to Paolo Poggio; paolo.poggio@ccfm.it
}

Received 3 May 2017; Accepted 9 May 2017; Published 21 August 2017

Copyright (c) 2017 Paola Songia et al. This is an open access article distributed under the Creative Commons Attribution License, which permits unrestricted use, distribution, and reproduction in any medium, provided the original work is properly cited.

In the article titled "Identification of Patients Affected by Mitral Valve Prolapse with Severe Regurgitation: A Multivariable Regression Model" [1], references [2,3] should have been included as references 24 and 25 in the original article.

Accordingly, in the introduction section, the text reading "Echocardiographically, MVP is defined as a single or bileaflet prolapse, at least $2 \mathrm{~mm}$ beyond the long-axis annular plane, while the assessment of valve regurgitation takes into account the effective regurgitant orifice area (EROA) [2]" should be changed to "Echocardiographically, MVP is defined as a single or bileaflet prolapse, at least $2 \mathrm{~mm}$ beyond the long-axis annular plane, while the assessment of the severity of MR relies on several parameters according to the current recommendations [24, 25]."

Additionally, the Acknowledgments section should be updated as follows:

"The authors thank the entire Cardiac Surgery Unit at Centro Cardiologico Monzino for the clinical data and blood collection. The authors also would like to thank Dr. Paola Gripari for patient evaluation and critical review. This work was supported by the Fondazione Gigi e Pupa Ferrari ONLUS and the Italian Ministry of Health [RC2015-BIO30-2613051 to Centro CardiologicoMonzino]."

\section{References}

[1] P. Songia, B. Porro, M. Chiesa et al., "Identification of Patients Affected by Mitral Valve Prolapse with Severe Regurgitation: A Multivariable Regression Model," Oxidative Medicine and Cellular Longevity, vol. 2017, Article ID 6838921, 6 pages, 2017.

[2] W. A. Zoghbi, D. Adams, R. O. Bonow et al., "Recommendations for noninvasive evaluation of native valvular regurgitation: a report from the American Society of Echocardiography developed in collaboration with the Society for Cardiovascular Magnetic Resonance," Journal of the American Society of Echocardiography: official publication of the American Society of Echocardiography, vol. 30, no. 4, pp. 303-371, 2017.

[3] R. A. Nishimura, C. M. Otto, R. O. Bonow et al., "2014 AHA/ ACC Guideline for the management of patients with valvular heart disease: executive summary: a report of the American College of Cardiology/American Heart Association Task Force on Practice Guidelines," Circulation, vol. 129, no. 23, pp. 2440 2492, 2014. 


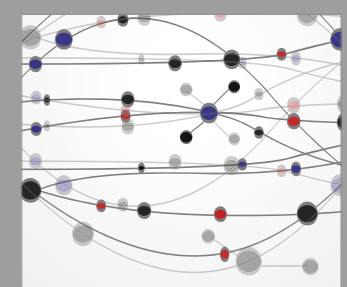

The Scientific World Journal
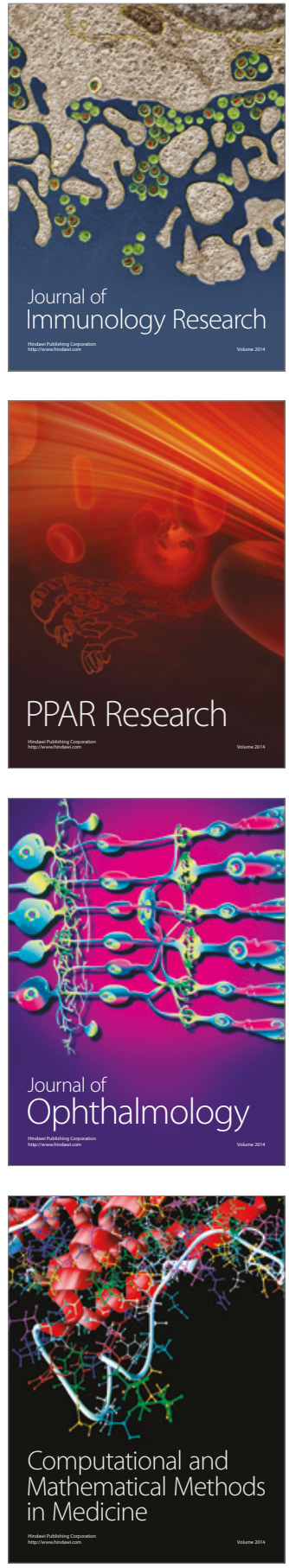

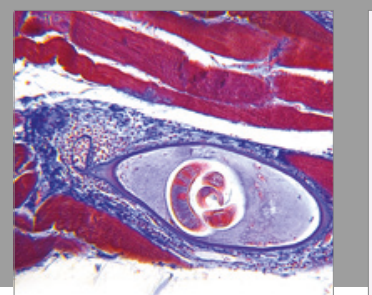

Gastroenterology Research and Practice
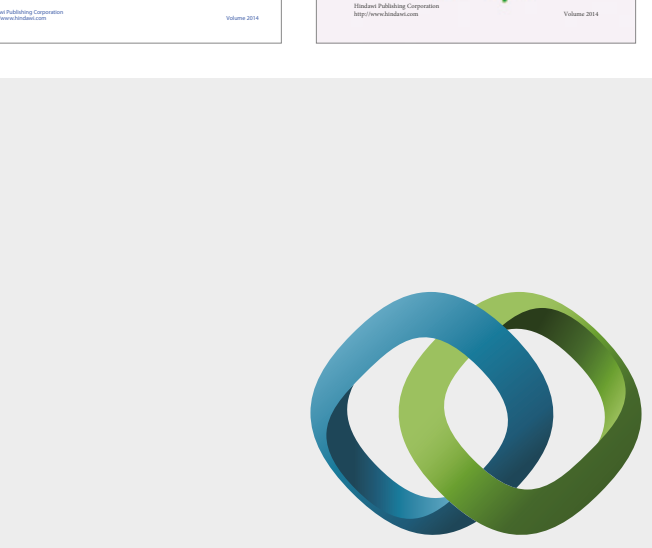

\section{Hindawi}

Submit your manuscripts at

https://www.hindawi.com
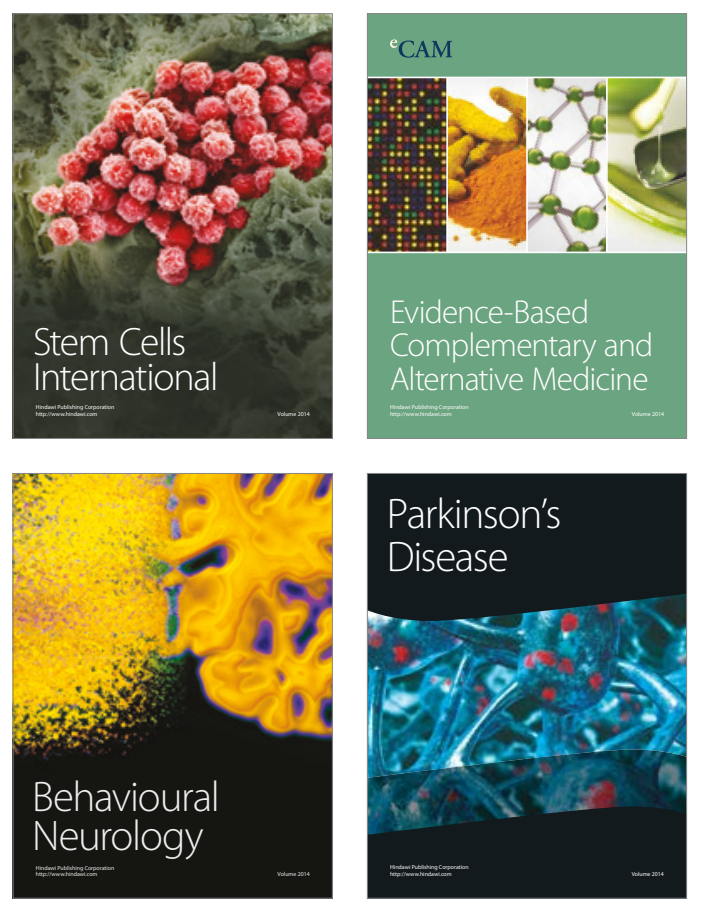
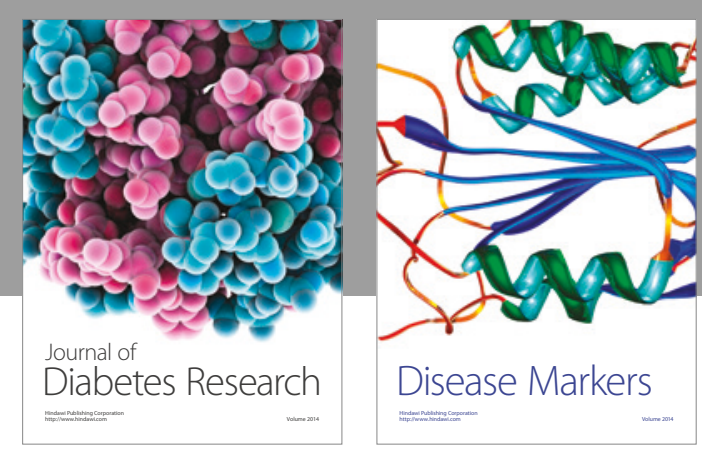

Disease Markers
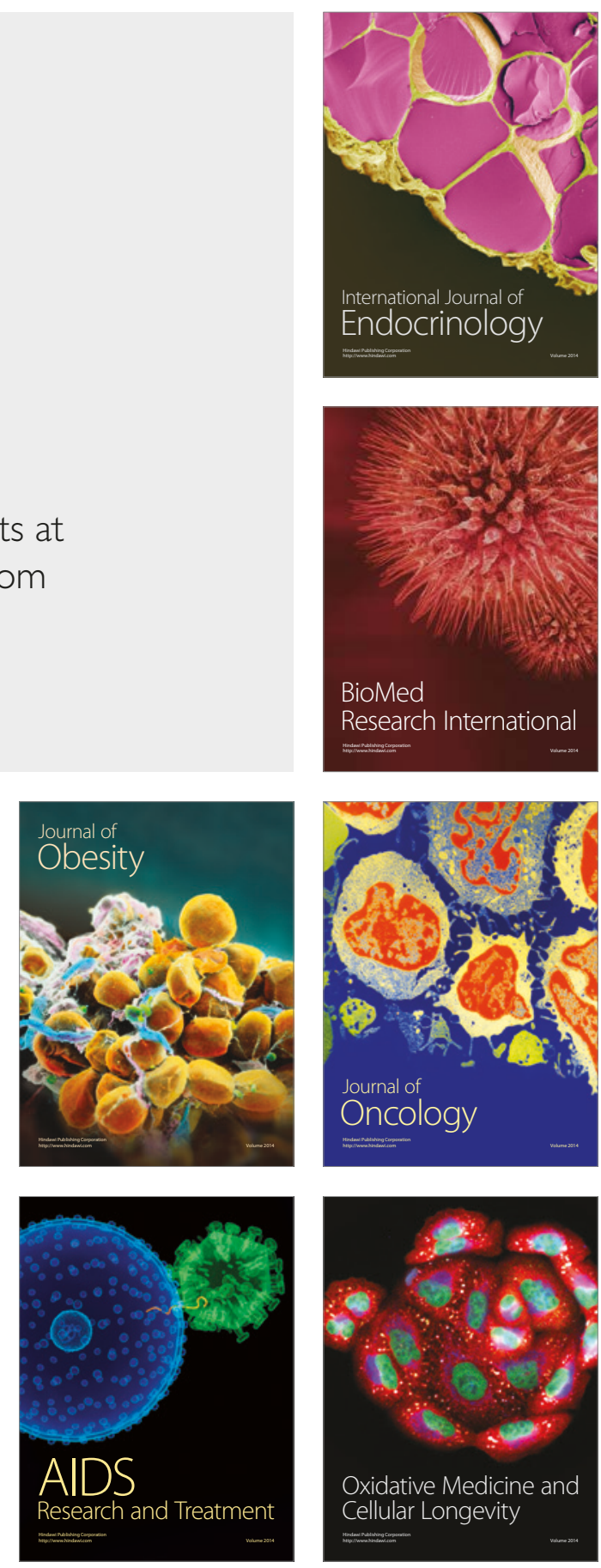